\title{
El síndrome $X$ frágil: identificación del fenotipo y propuestas educativas
}

\author{
Identification of the fragile $\mathrm{X}$ syndrome: \\ phenotype and educational approaches
}

\section{Resumen}

Las investigaciones confirman que el síndrome X frágil es la primera causa de discapacidad intelectual de tipo hereditario, pero aún es muy desconocido por profesionales de la educación, de la salud y de los servicios sociales. Una detección precoz evitaría la transmisión de la alteración genética y permitiría el establecimiento de las intervenciones más adecuadas para cada persona. En los últimos años se han dado grandes avances en la comprensión de este trastorno del neurodesarrollo. En este artículo se ha realizado una exhaustiva revisión de los estudios y contribuciones más significativas que existen hasta el momento acerca de este síndrome, de su interacción molecular, cerebral, cognitiva y conductual, de herramientas para hacer un primer cribado para la detección de posibles afectados y de sugerencias para la intervención, pero se necesita seguir avanzando para mejorar la evaluación e intervención en cada caso concreto.

Palabras clave

Discapacidad intelectual, intervención educativa, alteración genética, gen FRMI, trastorno del neurodesarrollo.

\begin{abstract}
Researchers confirm that fragile $\mathrm{X}$ syndrome is the leading cause of hereditary intellectual disability, but it is still largely unknown by educational professionals, health professionals and social service workers. An early detection could avoid transmission of the genetic disorder and would enable the establishment of the most appropriate interventions for each person. Great progress has been made over the last years in the understanding of the neurodevelopmental disorder. In this article, an extensive review about the most relevant studies and contributions that have been made so far concerning its molecular, brain, cognitive and conceptual interactions, the tools to sift in order to detect possible affected people, and also some tips on interventions. Nevertheless there should be continued progress in order to improve the assessment and the assistance in each particular case.
\end{abstract}

\section{Keywords}

Intellectual disability, educational intervention, genetic disorder, FRMI gene, neurodevelopmental disorder.

\section{Begoña Medina Gómez \\ <bmedina@ubu.es>}

Facultad de Educación.

Universidad de Burgos
Para citar:
Medina Gómez, B. (20I4): "El
síndrome X frágil: identificación del
fenotipo y propuestas educativas",
Revista Española de Discapacidad, 2
(2): $45-62$.
<http://dx.doi.org/I0.5569/2340-
5 $104.02 .02 .03>$

Fecha de recepción: 08-05-20I4 Fecha de aceptación: 05 -I I-20I4 


\section{Introducción}

En los últimos años, la gran mayoría de artículos que tratan sobre la discapacidad intelectual han agrupado a las personas por el grado de discapacidad, aunque cada vez son más los investigadores que analizan cuidadosamente los diversos síndromes genéticos, ya que en cada uno el funcionamiento del cerebro parece ser diferente.

Los avances de las últimas décadas en genética molecular, en neurociencia cognitiva, en neuropsiquiatría y en el estudio de la fisiología cerebral han permitido impulsar la idea de que cada síndrome presenta un complejo perfil que determina la aparición de puntos débiles y fuertes en la conducta de estas personas (Cornish et al., 2007; Kittlerof et al., 2008). Estos hallazgos ayudan en el diagnóstico clínico y en la búsqueda de intervenciones educativas que reconozcan las fortalezas y desafíos de cada trastorno en las principales etapas del desarrollo (Cornish et al., 2010).

El síndrome X frágil es la patología del neurodesarrollo hereditaria más frecuente y la segunda causa reconocida de discapacidad intelectual después del síndrome de Down. Se denomina así por la fragilidad del cromosoma $\mathrm{X}$ y se debe a la existencia de una porción de ADN anormalmente metilada y de mayor tamaño que se amplia de una generación a otra. Dicha metilación afecta al gen FMR I situado en el brazo largo del cromosoma X (Xq 27.3). La consecuencia es la ausencia de la proteína fmrpi, ausencia responsable del fenotipo que caracteriza a las personas con el síndrome, dadas las importantes funciones de esta proteína en el sistema nervioso central. Todavía hay que realizar más investigaciones que exploren cómo la falta de proteína repercute en la estructura y funcionamiento del cerebro. Hasta ahora, los estudios que han utilizado las técnicas de neuroimagen para conocer la estructura cerebral de las personas con síndrome $\mathrm{X}$ frágil han podido apreciar una disminución en el vermis cerebeloso y un aumento del núcleo caudado, del tálamo y del hipocampo (Eliez et al., 200I; Kates et al., I997; Mostofsky et al., 1998). En estas particularidades cerebrales observadas podría estar la explicación de las características específicas que estas personas presentan en el procesamiento cognitivo, en la capacidad visoespacial y en las funciones ejecutivas (Rivera et al., 2002).

Todos estos avances se han producido en los últimos setenta años. Los primeros estudios que mencionan este síndrome se remontan al año I943, cuando Martin y Bell describieron los primeros casos. En I969, se detecta el primer marcador diagnóstico a través del estudio del cariotipo en un medio de cultivo de células pobre en ácido fólico y que relacionan la enfermedad con una fragilidad localizada en el cromosoma X (Lubs, I969). En el año I99I, se descubre el gen responsable del síndrome y la expansión del triplete repetido CGG (Citosina, Guanina, Guanina) (Fu et al., I99I). Este descubrimiento permitió un mejor diagnóstico utilizando técnicas como la hibridación de Sothern-Blot (determina el estado de metilación y calcula el tamaño de la mutación) y la Reacción en Cadena de la Polimerasa PCR (permite determinar el número de repeticiones CGG) (De Diego, 20I4; Verkerk et al., I99I).

Desde la identificación de los primeros casos se han realizado estudios y publicaciones que han ido profundizando en el conocimiento del síndrome X frágil, pero aún quedan muchos aspectos de este trastorno que siguen siendo desconocidos o que requieren de una mayor profundización para la población general, incluso para los profesionales del ámbito sociosanitario y educativo. Este desconocimiento ha llevado a que haya afectados y portadores que ignoran que lo son y un alto porcentaje de adultos $(80 \%)$ sigue sin ser diagnosticado. Conocer las peculiaridades del síndrome facilita la identificación precoz de afectados y el establecimiento de las intervenciones multiprofesionales apropiadas para cada persona y su familia en los diferentes aspectos de su vida: sanitario, educativo, social, etc. La detección certera y precoz permite, entre otros beneficios: el establecimiento de programas de intervención más adecuados a cada persona; la prevención de trastornos 
físicos, afectivos y conductuales asociados al síndrome; la facilitación de apoyos y recursos adecuados para el desarrollo cognitivo de cada afectado; la aceptación y formación de la familia y educadores; todo ello redunda, en una mejora de la calidad de vida de los implicados, así como, poder romper la cadena de transmisión de la mutación.

El objetivo de este artículo es divulgar información actualizada sobre el estado actual del síndrome X frágil y facilitar herramientas o listas de verificación que han demostrado, en diferentes estudios, su idoneidad para realizar un primer cribado de posibles afectados por esta alteración genética y proporcionar orientaciones eficaces para el tratamiento de estas personas. Para ello, se han consultado las publicaciones que en los últimos diez años se han editado en nuestro país y artículos publicados en revistas internacionales con impacto.

\section{Manifestaciones en función de la secuencia genética, sexo y edad}

El síndrome $X$ frágil se caracteriza por una gran variedad de manifestaciones biológicas, neurocognitivas, conductuales y sociales diferentes para cada persona en función del número de tripletas, sexo y edad. Este hecho constituye un reto, no solo para el diagnóstico sino a la hora de abordar una intervención (De Diego, 20I4; Rosso y Darnells, 20I2).

En la actualidad se acepta que la secuencia genética relacionada con el síndrome $\mathrm{X}$ frágil se presenta en tres formas dependiendo del número de tripletas CGG: normal (entre 6-55 repeticiones), premutación (55-200) y mutación completa (expansiones superiores a 200 , por lo general, entre ı000 y 2000).

En los individuos premutados el funcionamiento del gen FMR I no llega a afectar lo suficiente como para que aparezcan síntomas del síndrome $\mathrm{X}$ frágil. Suelen tener niveles más reducidos de proteína fmrpi, pero en cantidad suficiente para que la madurez neuronal sea adecuada (Martínez, 2006). En el caso de las mujeres portadoras no muestran alteraciones fenotípicas relacionadas con el síndrome, aunque presentan una alta probabilidad ( $50 \%)$ de tener hijos afectados en la siguiente generación (GloverLópez y Guillen, 2006; Mínguez et al., 2009). En cambio en los varones premutados, aproximadamente un $20 \%$, son física, comportamental e intelectualmente normales y transmitirán el gen al ı००\% de sus hijas.

Como el síndrome es un desorden ligado al cromosoma X lo padecen fundamentalmente varones y es transmitido principalmente por mujeres. Las mujeres muestran un desarrollo muy variable con anomalías cuantitativas y cualitativas menos severas que los hombres, ya que al presentar dos cromosomas X compensan con uno los problemas funcionales del otro (Fernández et al., 2010). La prevalencia estimada del síndrome en la población española es de I/2500-4000 varones, I/4000-8000 mujeres y una prevalencia de portadoras de I/250 (Fernández-Carvajal et al., 2009). Estos datos coinciden con la frecuencia estimada en estudios realizados en otros países (Hagerman, 2008; Song et al., 2003).

La mayoría de los recién nacidos no presentan una apariencia física que haga sospechar de la existencia del síndrome. Las primeras dudas se inician en la infancia cuando los padres comienzan a percibir dificultades para caminar, aleteos, mordedura de manos, retraso en la adquisición del habla, falta de control de impulsos, rasgos autistas e hiperactividad (Fernández et al., 20ıо; Ferrando y Puente, 2008; Franco, 20I4). Cuando los padres detectan estas primeras dificultades acuden al especialista buscando respuestas a sus sospechas. $\mathrm{Si}$ en ese momento, se realizan pruebas de neuroimagen, en niños de I a 3 años, no se observan alteraciones neuroanatómicas que años más tarde si se constatan, lo que parece indicar que son cambios evolutivos asociados a la falta de proteína fmrpi (Hoeft et al., 20I0). Encontrar síntomas significativos antes del año es infrecuente en personas sin antecedentes 
familiares de discapacidad intelectual. Lo más habitual es que el diagnóstico se retrase hasta la adolescencia, sobretodo en niñas, ya que los síntomas físicos más significativos acostumbran a presentarse después de la pubertad y es en esta etapa, cuando se observan de forma clara dificultades cognitivas y adaptativas (ArtigasPallares, 20I I; Fernández et al., 20I0, Ferrando et al., 2004; Ferrando y Puente, 2008). Es frecuente que estos niños sean remitidos para valoración psicopedagógica o comiencen a recibir atención temprana sin diagnóstico o con diagnósticos genéricos de trastorno del espectro autista (TEA) o trastorno generalizado del desarrollo (TGD).

\section{Características del fenotipo físico y cognitivo-conductual}

Todas las características del fenotipo que definen un síndrome no tienen por qué aparecer en todas y cada una de las personas diagnosticadas, ni siquiera con la misma intensidad o en el mismo momento del desarrollo. Tampoco todos los individuos diagnosticados con el síndrome cumplen todos los criterios para su clasificación como persona con la alteración genética. Lo que el fenotipo define son los rasgos más frecuentes y significativos que suelen aparecer, pero rara vez un trastorno genético provocará una particular conducta en todas las personas afectadas (BrunGasca, 2006; Hodapp y Dykens, 2004). Por otro lado, puede suceder que un mismo síntoma sea característico de dos o más síndromes genéticos diferentes. Lo que se puede asegurar, a partir de los estudios realizados, es que la existencia de un síndrome genético predispone a los afectados a mostrar ciertas características y conductas específicas tanto de carácter físico, como conductual o cognitivo.

\subsection{Fenotipo físico y manifestaciones clínicas}

Los principales rasgos característicos que definen el fenotipo físico de varones con la mutación completa se concretan en rasgos faciales relevantes, problemas oftalmológicos, otitis media recurrente, displasias del tejido conjuntivo, escoliosis, afectaciones cardiocirculatorias, macroorquidismo pospuberal, convulsiones y alteraciones del sueño.

\section{Cuadro 1. Manifestaciones clínicas y fenotipo físico}

- Dismorfias faciales: macrocefalia, cara alargada y estrecha, fisuras palpedrales estrechas, frente prominente, boca grande con labios gruesos y labio inferior evertido, orejas grandes, prognatismo, paladar ojival con maloclusión dental.

- Alteraciones oftalmológicas: defectos de refracción y estrabismo.

- Otitis media recurrente y sinusitis

- Alteración del tejido conectivo: hiperlaxitud articular, hipotonía, torpeza motora, pies planos; línea de Sídney, hiperlasticidad cutánea, escoliosis

- Afectaciones cardiocirculatorias: prolapso de la válvula mitral y dilatación aórtica.

- Alteraciones endocrinas: Macroorquidismo pospuberal.

- Convulsiones.

- Alteraciones del sueño: insomnio y apneas obstructivas.

Fuente: Elaboración propia a partir de Barrio et al. (2006).

Los varones con la premutación no suelen presentar alteraciones morfológicas significativas aunque en edades avanzadas, a partir de la quinta-sexta década de vida, en el $20-45 \%$ de los casos, se ha descrito el síndrome de TemblorAtaxia (FXTAS). Esta patología se caracteriza por ataxia o dificultad para andar, temblores intencionales y una generalizada atrofia cerebral (demencia subcortical frontal) (FernándezCarvajal, 2014; Garber et al., 2008; Greco et al., 2002; Ramos, 2006; Toft et al., 2005).

Las manifestaciones clínicas expuestas en el caso de los hombres también aparecen en mujeres con mutación completa, aunque por lo general, son más leves y variables que en los varones. La 
sintomatología más característica, en el I 6-30\% de mujeres premutadas, es el riesgo de presentar Insuficiencia Ovárica Primaria (FXPOI), en la que los ovarios dejan de funcionar antes de los 40 años y aparece amenorrea (Fernández-Carvajal, 20I4; Mallolas et al., 2000; Rodríguez-Revenga et al., 2009; Tejada et al., 2008).

\subsection{Fenotipo cognitivo-conductual}

En un $30 \%$ de los casos, los rasgos físicos no están presentes o son poco evidentes. Por el contrario, la presencia de características del fenotipo conductual, cognitivo y lingüístico permite sospechar el diagnóstico en casi todos los afectados con el síndrome ya que, según Artigas-Pallares (20II), tienen un fuerte condicionamiento genético.

Un $80-90 \%$ de varones afectados presenta discapacidad intelectual de tipo moderado o severo, el Io-20\% restante es leve e incluso puede tener un cociente intelectual (CI) normal aunque, en estos casos, aparecen algunas dificultades de aprendizaje. Además, el grado de discapacidad guarda relación directa con el número de tripletas de CGG (Bourgeois et al., 2009). Una década de investigaciones ha demostrado que el fenotipo cognitivoconductual de una persona con el síndrome X frágil no se define, únicamente, por el grado de discapacidad intelectual, sino más bien por un perfil único de fortalezas y dificultades. Por lo que conocer el perfil de la persona permite adecuar las situaciones de aprendizaje para conseguir los mejores resultados.

Recientes estudios sostienen que los déficits cognitivos y conductuales observados en el síndrome X frágil están relacionados con una disfunción en estructuras dorsolaterales prefrontales y en los circuitos orbitofrontales. Estos hallazgos confirman que el núcleo central del perfil cognitivo en este síndrome se explica por déficits en las funciones ejecutivas (Peng et al., 2013; Van der Molen et al., 2010). Estas funciones son las responsables, entre otras, de regular la conducta, de cambiar de estrategia de forma flexible, de la inhibición de distracciones o de conductas inadecuadas. Estos aspectos se corresponden con las características más disfuncionales que se han descrito a nivel ejecutivo en el síndrome X frágil: dificultades de inhibición de estímulos irrelevantes; escasez de flexibilidad cognitiva; déficits en la resolución de problemas; limitaciones en la capacidad de la memoria de trabajo; dificultades visoespaciales; perseveración de actos motores y lingüísticos; y errores en el procesamiento abstracto (Cornish et al., 2005; Garber et al., 2008; Westmar y Maller, 2007).

\section{Cuadro 2. Fenotipo cognitivo conductual}

- Discapacidad intelectual.

- Déficits en funciones ejecutivas (planificación, abstracción, habilidades visoconstructivas, resolución de problemas, comprensión de reglas, razonamiento abstracto, etc.).

- Problemas de atención: selectiva (focalizarse en los importante e ignorar el resto de estímulos), alternante (cambiar el foco de un estímulo a otro) y dividida (capacidad para responder simultáneamente a dos estímulos o tareas).

- Hiperactividad e impulsividad.

- Dificultades de integración sensorial.

- Trastorno de ansiedad.

- Conductas autísticas (aleteos de manos, estereotipias) y defensibilidad sensorial (evitación del contacto físico y visual).

- Trastornos del lenguaje y la comunicación.

Fuente: Elaboración propia.

Estos pacientes van a experimentar muchas dificultades para responder a un estímulo concreto, ya que no pueden dejar de prestar atención al resto y no tienen clara la jerarquía de importancia de los estímulos. Este hecho dificulta la concentración en la tarea que se esté realizando. En un estudio llevado a cabo por Munir et al. (2000) se compararon las puntuaciones obtenidas por jóvenes varones con X frágil y jóvenes varones sin discapacidad en pruebas que evalúan los distintos tipos de atención. Los resultados hallados establecen que los primeros puntúan peor en atención selectiva, en atención dividida y en inhibición de estímulos 
distractores irrelevantes, y especialmente en la capacidad de planificar y organizar la búsqueda y desviar la atención de un estímulo a otro. En cambio, en el tiempo de respuesta en tareas de atención sostenida no fueron significativamente más lentos. Estas conclusiones son coincidentes con las obtenidas en estudios con adultos varones con $\mathrm{X}$ frágil, en los que se observa un rendimiento relativamente intacto en tareas de atención sostenida, pero con limitaciones en aquellas que requiere cambio atencional, si se les compara con las respuestas de personas con discapacidad intelectual de etiología no filiada (Hagerman y Cronister, I996).

Las dificultades de atención suelen ir asociadas a impulsividad e hiperactividad, lo que se conoce como la triada de la hiperactividad, y que se manifiesta al menos en el 50-80\% de los varones con el síndrome X frágil, siendo más frecuente que en otros grupos de personas con discapacidad. En estas personas la capacidad de autorregulación del comportamiento es muy escasa y la complejidad $\mathrm{o}$ incapacidad para mantener la atención sobre una tarea, si esta dura demasiado tiempo, puede provocar excitación y estallidos conductuales como forma de descarga de la ansiedad, especialmente si el entorno se caracteriza por estar excesivamente sobrecargado de estímulos (Baumgardner et al., I995; Medina-Gómez, 20I4). Según Barrio et al. (2006) es el problema conductual más frecuentemente asociado al síndrome, con una etiología precisa y una evolución o pronóstico diferentes en cada caso.

En esta línea, algunos estudios encuentran que entre un $30-70 \%$ de las personas con síndrome $\mathrm{X}$ frágil puede presentar trastornos de ansiedad. Esta tasa es, según algunos autores, superior en un 3,I \% de la observada en personas con discapacidad intelectual con diferente etiología (Cooper et al., 2007) y un $8,9 \%$ mayor que en la población general (Martín-Pérez et al., 2003). Las consecuencias del nivel de discapacidad de estas personas dificultan, o incluso impiden, que expresen el malestar o distrés propio de un trastorno de ansiedad. Lo más habitual es que lo manifiesten en forma de síntomas y signos motores o fisiológicos que caracterizan, en parte, el fenotipo conductual (Cornish et al., 200I).
Los síntomas se presentan principalmente ante situaciones nuevas, con excesivos estímulos (ruidos, personas), con un nivel de exigencia que supera el nivel de competencia de la persona y en momentos de transición o cambio de actividad (Symons et al., 2010). Estas conductas tienden a mejorar a medida que se incrementa la edad, pero en muchos casos, son mal interpretadas por profesionales que no conocen las dificultades de integración sensorial que presentan estas personas.

Como consecuencia, pueden ver limitadas las oportunidades para participar en distintos contextos, interferir significativamente en la adquisición de nuevos aprendizajes, e incluso pueden llegar a ser catalogadas como agresivas y recibir tratamiento psicofarmacológico por ello. Para evitar estas consecuencias es necesario efectuar un análisis funcional que permita identificar qué está provocando el aumento de la ansiedad (estresores) y qué es lo que precipita la aparición de estas conductas (factores desencadenantes). Son numerosos los condicionantes ambientales que pueden actuar como estresores o desencadenantes de la alteración conductual (Novell y Estrada, 20I4). Por otro lado, parece ser que una alteración en la función hipotalámica-hipófisis-adrenal puede estar relacionada con el elevado grado de ansiedad y la hiperactividad de los pacientes con el síndrome X frágil (Wisbeck et al., 2000).

Las personas con el síndrome $\mathrm{X}$ frágil prefieren observar en lugar de participar activamente en la realización de actividades, incluso se pueden mostrar incómodos ante saludos, cumplidos, críticas o al ser el centro de la atención, ya que no pueden procesar estas situaciones de forma adecuada (Artigas-Pallares, 20I I). Este procesamiento inadecuado entorpece la organización, integración y entendimiento de estímulos (auditivos, táctiles o visuales) y provoca la hipersensibilidad a sonidos (ruidos, gritos, exclamaciones verbales en voz alta) y a manifestaciones muy afectuosas (abrazos, besos, etc.). Todo esto les produce estrés y les generan conductas reactivas de tipo autístico (aleteos o mordedura de manos, estereotipias, gritos o rabietas) y de defensibilidad sensorial (defensa táctil y evitación del contacto visual). 
Las conductas de defensibilidad se manifiestan a través de gestos esquivos, distanciamiento físico, situarse en lugares apartados, apartar la cara en las interacciones sociales, etc., que aparecen principalmente ante estímulos nuevos e imprevistos, cuando se incide en zonas del cuerpo sensibles o si el paciente está en contacto con texturas que pueden resultar especialmente molestas. Estas conductas son la manera que tienen las personas con $\mathrm{X}$ frágil de afrontar las situaciones en las que sienten que hay un exceso de información y su ejecución les ayuda a tolerar más fácilmente las características del entorno. Con el objetivo de reducir estas conductas se utiliza la dieta sensorial que tiene como finalidad que la persona consiga y mantenga un estado de activación apropiado durante el día (Wilbarger y Wilbarger, 2002).

Las características del lenguaje y de la comunicación en este colectivo presentan una especificidad en todas las áreas del lenguaje, con un inicio tardío que se va robusteciendo con el desarrollo fonológico y la adquisición de vocabulario, en cambio las dificultades morfosintácticas y pragmáticas persisten (DiezItza et al., 20I4).

Las características del fenotipo cognitivoconductual femenino, en general, están menos estudiadas por el menor nivel de afectación y de prevalencia en las mujeres. Algunas investigaciones indican que aproximadamente el $70 \%$ de las mujeres con mutación completa presenta déficit cognitivo de tipo límite (Cornish et al., 2005). Otras, por el contrario, recogen que, aunque el $60-70 \%$ tenga un coeficiente intelectual normal, la mayoría tienen: trastornos del lenguaje; trastornos de lectoescritura; déficits de atención e hiperactividad; trastornos emocionales; bajo rendimiento en matemáticas; estereotipias y conductas autistas; limitaciones en la toma de decisiones; organización de la información; autorregulación de la conducta (Ferrando et al., 2004; Ferrando et al., 2008; Warren y Sherman, 200I), debido a las disfunciones presentes en la corteza prefrontal y parietal (Cornish et al., 2005; Menon et al., 2004). El tener un nivel intelectual, en general, superior a los varones, facilita que las primeras adquisiciones del desarrollo no se retrasen, por lo que consiguen un mayor nivel en los aprendizajes escolares y las dificultades en la escuela suelen aparecer más tarde y acostumbran a ser menos graves que en los varones. Se debe tener en cuenta, que estas dificultades son similares pero menos acentuadas en las mujeres portadoras.

Las principales características conductuales en las mujeres están relacionadas con la timidez y las dificultades en las relaciones sociales, sobre todo para hacer y mantener relaciones de amistad, lo que las conduce a situaciones de aislamiento social por evitación. Se han relacionado estas dificultades con un déficit en la comprensión e interpretación de contextos sociales y en la interacción con los otros. En ocasiones hacen lecturas erróneas de las intenciones de los demás, provocando desagradables situaciones de malentendidos y también tienen dificultad para comprender los puntos de vista de otras personas (Brun-Gasca, 2006).

En las personas con síndrome $\mathrm{X}$ frágil se ha constatado que, junto a las dificultades comentadas, presentan buenos niveles de competencia en el procesamiento simultáneo, en el reconocimiento visual, en la comprensión de los principios globales, en la utilización de materiales concretos y tangibles, en la utilización de mapas y planos, siempre que todo el material se presente de manera conjunta (Kaufman et al., 2004).

\section{Síndrome $X$ frágil y trastorno del espectro autista}

El 24-33\% de los varones con síndrome X frágil cumple criterios para el diagnóstico de autismo (Artigas-Pallares, 2006; Bayley et al., 200I; Turk, I995) y casi todos los diagnosticados con el síndrome presentan síntomas autísticos como estereotipias, perserveración, aleteos, conductas de evitación, defensa táctil (Artigas-Pallares et 
al., 200I; Hagerman, 2004). Así mismo, del 2 al $6 \%$ de personas con autismo tiene el síndrome X frágil (García-Nonell et al., 2006; Kaufmann et al., 2004; Reddy, 2005), según ArtigasPallares (20I I) es la causa genética específica más frecuente hallada en el autismo.

Estudios recientes ponen de manifiesto que, aunque ambos grupos presenten un perfil conductual muy similar, existen matices diferenciales. Un ejemplo de ello es que las personas con síndrome $\mathrm{X}$ frágil, aunque manifiesten dificultades en el manejo de las relaciones sociales, éstas no les son indiferentes ni tampoco las rechazan, sino que incluso las provocan y las buscan de forma activa (McDuffie et al., 20I0). Si bien evitan el contacto ocular, su mirada es comunicativa y no parece estar motivada por falta de conciencia social, sino por la hiperexcitabilidad y ansiedad social provocada por las interacciones sociales (Cornish et al., 2010; Hall et al., 2009).

\section{Herramientas para la identificación del fenotipo conductual y cognitivo}

Una cuidadosa observación del comportamiento puede, por si misma, sugerir el diagnóstico del síndrome, sin olvidar la gran variabilidad de características fenotípicas que pueden presentar los afectados. El uso de listas de los síntomas más frecuentes permite incrementar la precisión de las sospechas y seleccionar a los pacientes a los cuales es necesario llevar a cabo el estudio genético para la confirmación del diagnóstico.

Hagerman et al. (I99I) elaboraron una herramienta, Checkilist de Hagerman, que consta de I 3 ítems y que aplicaron a I07 varones (Cuadro 3). Las puntuaciones iguales o superiores a I9 confirmaron, tras la realización del estudio genético, que el $60 \%$ presentaba el síndrome X frágil. Si la puntuación está

\section{Cuadro 3. Checklist de Hagerman}

\begin{tabular}{|c|c|c|c|}
\hline & $\begin{array}{c}\text { No presente } \\
0\end{array}$ & $\begin{array}{c}\text { Dudoso o borderline } \\
1\end{array}$ & $\begin{array}{c}\text { Presente } \\
2\end{array}$ \\
\hline \multicolumn{4}{|l|}{ Discapacidad intelectual } \\
\hline \multicolumn{4}{|l|}{ Hiperactividad } \\
\hline \multicolumn{4}{|l|}{ Dificultades de atención } \\
\hline \multicolumn{4}{|l|}{ Defensa táctil } \\
\hline \multicolumn{4}{|l|}{ Aleteos de manos } \\
\hline \multicolumn{4}{|l|}{ Mordida de manos } \\
\hline \multicolumn{4}{|l|}{ Dificultad en el contacto ocular } \\
\hline \multicolumn{4}{|l|}{ Habla repetitiva } \\
\hline \multicolumn{4}{|c|}{ Hiperextensibilidad. Articulación metacarpo-falángica } \\
\hline \multicolumn{4}{|l|}{ Orejas grandes y/o prominentes } \\
\hline \multicolumn{4}{|l|}{ Macroorquidismo } \\
\hline \multicolumn{4}{|l|}{ Surco simiesco o línea de Sydney } \\
\hline Historia familiar de discapacidad intelectual & & & \\
\hline
\end{tabular}

Fuente: Hagerman et al. (I99I). 
comprendida entre I6-I9 puntos, el diagnóstico se confirma en el $45 \%$ de los casos. La aplicación de la Checklist de Hagerman permite un primer cribado ante puntuaciones superiores a 16 puntos, pero se aconseja un estudio molecular en el que se constate el número de repeticiones CGG para confirmar el diagnóstico.

Posteriormente se propuso una versión abreviada de la herramienta a 6 ítems que no reduce la sensibilidad (Cuadro 4), en la que una puntuación igual o mayor a 5 justifica la solicitud del estudio genético (Grangreco, I996). Maes et al. (2000) proponen un cuestionario de 28 ítems que deben ser contestados con "si" o “no" (Cuadro 5). Una puntuación total superior a I7 recomienda la realización de estudios moleculares para la confirmación del diagnóstico de síndrome X frágil.

En un estudio llevado a cabo en nuestro país, en centros de adultos con discapacidad intelectual, para detectar varones afectados por el síndrome X frágil, García-Alonso et al. (2008) concluyeron que el protocolo HRD-2 es útil para la realización de un primer cribado que permita la identificación de personas susceptibles de presentar la mutación (este instrumento incluye en el último apartado la Checklist de Hagerman) (ver Cuadro 6).

\section{Cuadro 4. Versión abreviada de Checklist de Hagerman}

\begin{tabular}{|c|c|c|c|}
\hline & 0 puntos & 1 punto & 2 puntos \\
\hline Cociente intelectual & $>85$ & $70-85$ & $<70$ \\
\hline $\begin{array}{l}\text { Historia familiar de discapacidad } \\
\text { intelectual }\end{array}$ & No aparece & Aparece & $\begin{array}{c}\text { Aparece y está ligada al } \\
\text { cromosoma X }\end{array}$ \\
\hline Cara alargada & No aparece & Moderada & Muy alargada \\
\hline Orejas grandes y hacia fuera & No aparece & Leve & Muy evidente \\
\hline Problemas de atención con hiperactividad & No aparece & Hiperactividad & $\begin{array}{c}\text { Hiperactividad y } \\
\text { problemas de atención }\end{array}$ \\
\hline Autismo y problemas de comportamiento & No aparece & $\begin{array}{l}\text { Aparece levemente } \\
\text { uno de los dos }\end{array}$ & $\begin{array}{l}\text { Aparece ambos o uno de } \\
\text { los dos muy marcado }\end{array}$ \\
\hline TOTAL & & & \\
\hline
\end{tabular}

Fuente: Grangreco (I996).

\section{Cuadro 5. Cuestionario de características del síndrome $\mathrm{x}$ frágil}

- Cara estrecha y alargada

- Frente amplia

- Mandíbula prominente

- Orejas grandes y bajas

- Macroorquidismo

- Hiperlaxitud de los dedos

- Hiperlaxitud articular (otras)

- Hiperactividad

- Sensibilidad sensorial exagerada

- Impulsividad

- Desorganización

- Timidez

- Necesidad de ayuda para actos simples

- Incoherencia afectiva
- Vergüenza

- Paso rápido de alegría a tristeza

- Hipersensibilidad hacia los cambios

- Mordeduras en las manos

- Movimientos de manos estereotipados

- Aleteo de manos y brazos

- Evitación del contacto ocular

- Ocultamiento de cara

- Defensa táctil

- Rapidez al hablar

- Hablador

- Perserverante

- Actos repetitivos

- Lenguaje propio

Fuente: Maes et al. (2000). 
Cuadro 6. Hoja de recogida de datos para la detección de varones con posible síndrome X frágil. HRD-2

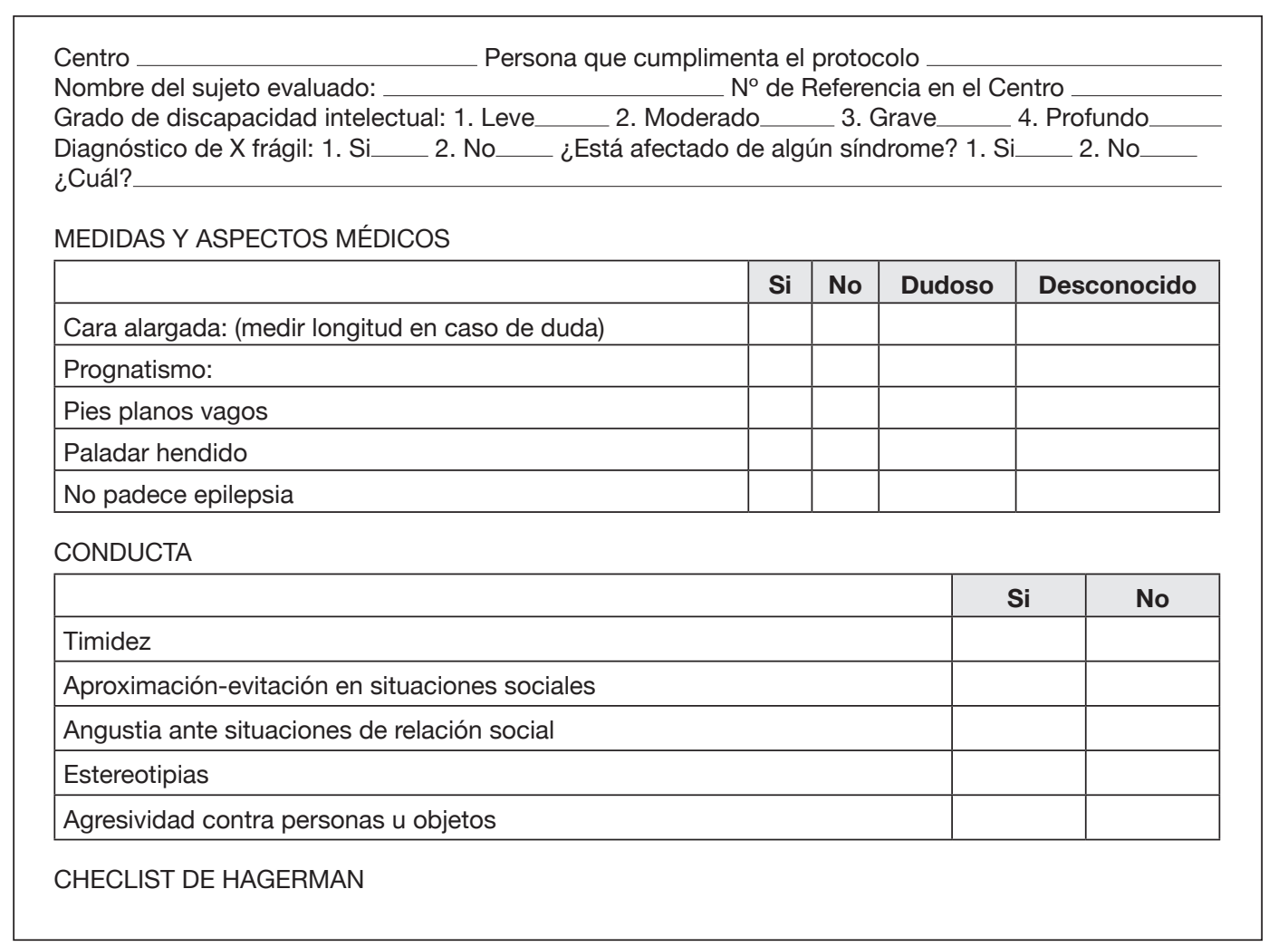

Fuente: Adaptada de García-Alonso et al. (2008).

\section{Importancia de la intervención}

Durante las últimas décadas ha habido un gran desarrollo, aunque no el suficiente, sobre el conocimiento de las causas y consecuencias de las alteraciones originadas por la mutación genética, pero existe una gran necesidad de divulgar y trasladar estos conocimientos a la intervención que deben realizar educadores, profesionales clínicos y familiares para conseguir estrategias de actuación óptimas y flexibles (Cornish et al., 2010).

Establecer intervenciones concretas para el colectivo de personas con la mutación completa o con la premutación es una tarea hasta el momento por lo menos compleja, debido a la gran variedad de sintomatología existente, la diversidad interindividual y los pocos estudios realizados sobre la eficacia de intervenciones llevadas a cabo. Estos obstáculos no deben ser una excusa para no tener actitudes entusiastas, ya que se pueden proponer acciones e intervenciones que pueden ser efectivas, siempre en función de cada persona y de las condiciones de los entornos donde se realizan.

En primer lugar es necesario diagnosticar el síndrome. La detección precoz es fundamental para llevar a cabo, lo antes posible, un consejo genético y dar el adecuado abordaje psicoeducativo a cada individuo en función de las características del fenotipo cognitivoconductual que presente. Posteriormente, se debe realizar una evaluación exhaustiva de las habilidades y competencias cognitivas, conductuales y lingüísticas de cada afectado 
para establecer el perfil de capacidades y limitaciones y, de este modo, establecer la intervención más adecuada. La intervención debe ser diseñada y aplicada por profesionales de distintas disciplinas. Con el entrenamiento y la facilitación de apoyos específicos, muchas de las limitaciones de las personas con síndrome $\mathrm{X}$ frágil son subsanadas, disminuidas o compensadas y la persona va tener un mejor funcionamiento que repercute en una mejor calidad de vida y una mayor participación social (Cohn et al., 2000).

\subsection{Tratamientos farmacológicos}

Hasta el día de hoy, no se conoce cura para el síndrome X frágil. Los tratamientos médicos se han utilizado para controlar o aliviar algunas características fenotípicas. Los pocos estudios realizados hasta el momento sobre la efectividad de los tratamientos farmacológicos no han obtenido resultados concluyentes. En algunas investigaciones se ha analizado cómo la medicación estimulante mejora los síntomas de hiperactividad, aunque en niños puede provocar un incremento de la irritabilidad y de conductas inadecuadas. En otras ocasiones se ha demostrado cómo la utilización de antipsicóticos, en pequeñas dosis, puede ayudar a reducir altos niveles de agresividad, de inestabilidad del estado de ánimo y controlar rabietas severas (Hagerman et al., 2009). En líneas generales, se puede decir que la medicación se utiliza para estimular el sistema nervioso, evitar los síntomas de hiperactividad, la impulsividad, la agresividad, las crisis epilépticas, la ansiedad y los comportamientos obsesivos-compulsivos (Berry-Kravis et al., 2004). En la actualidad se están llevando a cabo investigaciones experimentales para comprobar la efectividad de compuestos que mejoren el aprendizaje y el comportamiento de los afectados (De Diego Otero et al., 2009; RomeroZerbo et al., 2009).

El uso de psicofármacos en el síndrome X frágil no es una alternativa a otras vías de intervención no farmacológica, sino que debe contemplarse como un complemento para optimizar las intervenciones psicológicas y pedagógicas (Artigas-Pallares, 2006). Hasta que un tratamiento específico para el síndrome sea efectivo, la propuesta más utilizada para tratar a estas personas pasa por una intervención multidisciplinar integrada, en la que todos los profesionales implicados en la normalización de los afectados trabajan de forma conjunta (De Diego-Otero, 20I4).

\subsection{Propuestas educativas. Intervenciones cognitivas y psicosociales}

El logro de una buena intervención es una tarea compleja, pero como se ha reflejado en este artículo, el conocimiento de las características del fenotipo de estas personas puede facilitar esta tarea. A continuación se exponen, brevemente, algunas propuestas de intervención que han sido planteadas por diferentes autores para la atención a personas con el síndrome X frágil, y que requieren de más investigación para conocer su idoneidad y eficacia.

- Proponer tareas que resulten atractivas que sean foco de interés de la persona y, progresivamente, ir aumentando los periodos de atención. Es conveniente ir introduciendo poco a poco estímulos nuevos y agradables para que la persona vaya adaptándose a las nuevas demandas e integrando la información que le llega por distintas vías sensoriales, y es importante permitir momentos de descanso entre tareas. El rechazo inicial que puede aparecer en estas personas, al tener que realizar tareas nuevas, no debe llevar a pensar que la persona no va a disfrutar de esa actividad, que no le gusta y llegar a la determinación de no intentarlo (Barrio et al., 2008; Medina-Gómez, 20I4).

- Establecer ambientes muy estructurados y conocidos para la persona (orden en el espacio físico, establecimiento de horarios fijos para actividades, rutinas diarias, interlocutores sociales seguros, etc.). Facilitar entornos predecibles permite saber qué y cómo comportarse al acotar de forma 
clara espacios, momentos, materiales y personas que van a formar parte de una actividad, y así poder anticipar lo que va a ocurrir (Medina-Gómez, 20I4).

- Entrenar a la persona a que seleccione los elementos significativos para la realización de una tarea con éxito, proporcionar información sobre a qué estímulos debe atender y cuáles debe ignorar. Hay que empezar por el control externo que le proporciona un adulto-profesional, para acabar con un autocontrol de la situación (Dagnan y Jahoda, 2006; Hatton, 2002).

- Reducir las distracciones que ocasiona una excesiva estimulación ambiental, tomando medidas como: situar a la persona de espaldas a la puerta; colocar cortinas en ventanas; poner en la mesa solo el material necesario para realizar la tarea; utilizar mobiliario blando y cómodo; trabajar en grupos reducidos y facilitar únicamente la información necesaria utilizando la vía visual (emplear material gráfico como fotos, dibujos, pictogramas, etc., la observación de la realización de la tarea por parte de iguales, etc.) (Novel y Estrada, 20I4).

- Realizar actividades físicas que impliquen desplazamientos (juegos, paseos, deportes, recados, traslado de objetos, etc.) y actividades relajantes (escuchar música, técnicas de respiración, etc.) para liberar la excesiva energía acumulada como consecuencia de las interacciones sociales y de situaciones con excesiva estimulación (Novel y Estrada, 20I4).

- Para controlar la sobreestimulación por exposición a ambientes con muchos estímulos, facilitar los traslados o los cambios de actividad se pueden usar protectores personales (gafas oscuras, viseras o gorras, ropa larga, amplia, suave y sin adornos, etc.) y llevar objetos personales (carpetas, libros, bolsos, pelotas, cojines, muñecos de pequeño tamaño, etc.). La estrategia a seguir será diferente de una persona a otra (Novel y Estrada, 20I4).
- Evitar lugares multitudinarios y, si no fuera posible, tolerar determinadas estrategias que le permitan el manejo de la ansiedad social, como por ejemplo, mantenerse distante del grupo, evitar el contacto físico y llevar algún objeto personal. Es importante anticiparle cuál es la situación a la que se va a enfrentar, indicarle claramente cómo actuar en esa circunstancia y dejarle un tiempo prudencial para que procese la información antes de pasar a la acción (Sean-Oon et al., 2008).

- Dar órdenes utilizando un tono de voz firme pero cálido y sereno, sin utilizar un estilo imperativo como "ven", "acércate”. Con la emisión de estas órdenes se provoca justamente el efecto contrario, el distanciamiento y la huida (Scharfenaker, I999).

- Como se ha comentado, en general estas personas tienen más facilidad para captar la información visual que la auditiva, aprenden por lo que ven hacer más que por las explicaciones que reciben de lo que se debería hacer. Esta cualidad cognitiva debe ser utilizada como método de entrada de información para favorecer el aprendizaje (Brun-Gasca, 2006).

- Permitir la observación de la realización de una tarea por otra persona en entornos naturales, con dos objetivos: evitar la tensión que aparece al tener que dar respuesta a demandas del ambiente y facilitar el aprendizaje utilizando la imitación, el procesamiento simultáneo y la vía visual, estrategias idóneas para la adquisición de conocimientos en personas con X frágil (Fernández et al., 2010).

- Permitir la elección personal y la toma de decisiones. Si fuera necesario, dar dos opciones para elegir en lugar de ofrecer múltiples alternativas. Para que la persona pueda hacer una buena elección, es importante volver a incluir opciones que ya se habían descartado (Medina-Gómez, 20I4). 
Como se puede observar, todas las pautas de intervención que se proponen en este artículo están interrelacionadas y pretenden ser una breve orientación para los profesionales del campo de la educación, de la salud y de los servicios sociales, ya que en muchos casos no se sabe cómo intervenir con las personas con el síndrome X frágil, sobre todo cuando presentan características fuera de lo habitual. La ejecución de tareas de las personas con el síndrome será relativamente mejor si se tienen en cuenta estos aspectos que pueden maximizar el aprendizaje y evitar la frustración que aparece al ofrecer tareas que superan el nivel de competencia. Por otro lado, podemos observar que algunas pautas que contribuyen a mejorar el rendimiento y la inclusión social de la persona con síndrome $\mathrm{X}$ frágil se concretan en adaptaciones y modificaciones de los entornos.

Para conseguir una intervención eficaz es esencial incluir a la persona en la toma de decisiones sobre los objetivos a trabajar y tener en cuenta las oportunidades que los contextos faciliten a la persona con discapacidad. Seguir investigando y formándose en la comprensión del fenotipo cognitivo y conductual redundará en un mejor aprovechamiento de sus capacidades, un mejor desarrollo del proceso de enseñanza-aprendizaje, un mejor rendimiento, una mayor autonomía, una mejor inclusión social, una disminución de la presencia de conductas inadecuadas y una buena calidad de vida. Es necesario que los educadores estén atentos a las necesidades de sus alumnos y sepan trabajar sus potencialidades, explorar sus preferencias y/o compensar aquellas limitaciones que por carencias estructurales pueden presentar.

\section{Conclusiones y perspectivas de futuro}

Existen características físicas, cognitivas y conductuales con alta prevalencia en personas con síndrome $\mathrm{x}$ frágil. Su conocimiento y estudio pueden contribuir a un diagnóstico precoz. Existen herramientas que han demostrado su efectividad para detectar las características fenotípicas más frecuentes en este trastorno del neurodesarrollo, pero es imprescindible la confirmación del diagnóstico con pruebas moleculares. Para Fernández-Carvajal y De Diego (20I4) el reto es el desarrollo de métodos rápidos y fiables de diagnóstico, que posibiliten el análisis de toda la población y su inclusión en los programas de cribado de todos los recién nacidos para de este modo detectar de forma precoz a los afectados. La detección temprana de la premutación o mutación del gen FMR I en una familia permite establecer las posibilidades de aparición de la mutación completa en una futura generación o el nacimiento de un niño con el síndrome.

Hasta que se encuentre un tratamiento médico efectivo para compensar la falta de proteína fmrpi y las consecuencias que esta carencia ocasiona a nivel cerebral y físico, la propuesta más adecuada para la intervención pasa por un abordaje multidisciplinar integrado. Los estudios demuestran que pacientes con esta alteración, si reciben un programa de estimulación adecuada y un apoyo psicopedagógico certero, presentan menos déficits cognitivos en la edad adulta (De Diego-Otero, 20I4). Seguir profundizando en el conocimiento de las relaciones que las alteraciones neurocerebrales tienen en los procesos cognitivos de estos pacientes y en las manifestaciones conductuales permitirá especificar sobre qué aspectos hay que incidir para conseguir el máximo desarrollo y capacitación de cada persona.

La obtención de pruebas sensibles para la identificación del perfil de capacidades y limitaciones mejorará la evaluación individual $y$, por lo tanto, el establecimiento de las intervenciones más idóneas a partir de las potencialidades y dificultades. Trabajar para conocer cuáles son los programas y acciones más adecuadas en este colectivo es esencial y debe ser una prioridad de cara al futuro. La existencia de diferentes estilos de aprendizaje y características comportamentales, lleva a sugerir cómo la educación especial puede ser efectiva, pero en la mayoría de los casos, los individuos con síndrome $\mathrm{X}$ frágil reciben los mismos servicios 
de educación especial y terapéuticos que están disponibles para cualquier otro niño y adulto con discapacidad intelectual (Losh et al., 2012).

Dada la gran variabilidad del colectivo, se necesita un estudio individual multidisciplinar que les permita la mejora de su potencial, de modo que sean integrados como miembros útiles de la sociedad. Por lo tanto, la intervención debe ir precedida por una evaluación multidisciplinar para la planificación de la atención educativa más adecuada a las necesidades emocionales y de comportamiento de los afectados, sin obviar los problemas médicos asociados que pueden presentar (Braden et al., I999). Se considera que las intervenciones tienen que ser específicas para cada sujeto, dada la gran variabilidad individual.

Y por último, y no por ello menos importante, se debe dar mayor divulgación y visibilidad al síndrome y velar por los derechos de los afectados. En este sentido, son muchos los esfuerzos que se están realizando para trabajar no solo para estas personas y sus familias, sino junto a ellas, pensando en cuáles son las demandas y necesidades de cada una de las personas con el síndrome X frágil. 
Referencias bibliográficas

Artigas-Pallares, J. (20I I): "Síndrome X frágil”. En: Artigas-Pallares, J. y Narbona, J. (eds.): Trastornos del neurodesarrollo, Barcelona: Viguera.

Artigas-Pallares, J. (2006): "El trastorno de déficit de atención/hiperactividad en la consulta del pediatra. Algunas sugerencias”, Revista Pediatría de Atención Primaria, VIII (4): II 5-I 33 .

Artigas-Pallares, J. et al. (200I): “Aspectos médicos y neuropsicológicos del síndrome X frágil”, Revista Neurología Clínica, 2(I): 42-54.

Barrio, J. A. et al. (2008): "Síndrome X frágil”. En: FEAPS: Síndromes y apoyos. Panorama desde el punto de vista de la ciencia y de las asociaciones, Madrid: FEAPS

Baumgardner, T. et al. (I995): "Specifications of the neurobehavioural associations in males with fragile X syndrome”, Pediatrics, 95: 744-752.

Bayley, J. A. et al. (200I): “Segmental Duplications: Organization and Impact Within the Current Human Genome Project Assembly", Genome Research, II: I005-IOI7.

Berry-Kravis, E. et al. (2009):“A pilot open label, single dose trial of fenobam in adults with fragile X syndrome", Journal Medical Genetic, 46: 266-27I.

Bourgeois, E. et al. (2009): “The pro-Th2 cytokine IL-33 directly interacts with invariant NKT and NK cells to induce IFN- $\gamma$ production", European Journal of Immunology, 39(4): 9I4I 75 (en línea). 〈doi:I0.I002/eji.200838575〉.

Braden-Harder, L. et al. (I999): “U.S. Patent $N^{o}$. 5,933,822”, Washington, DC: U.S. Patent and Trademark Office.

Brun-Gasca, C. (2006): "El fenotipo cognitivoconductual”. En: Tejada M. I. (dir.): Sindrome $X$ Frágil. Libro de consulta para familias y profesionales, Madrid: Real Patronato sobre Discapacidad (en línea). ‘http://www.fraxa.org/ pdf/librosxf.pdf.
Cohn, E. et al. (2000): "Parental hopes for therapy outcomes: children with sensory modulation disorders", American Journal Occupational Therapy; 54: I-8.

Cooper, W. W. et al. (2007): Data envelopment analysis. A Comprehensive Text with Models, Applications, References and DEA-Solver Software, New York: Springer Science Business Media, LLC.

Cornish, K. M. et al. (2010): "Fragile X syndrome and associated disorders". En: Holmes, J. (ed.): Advances Children Developmental Behavior, London: Elsevier.

Cornish, K. M. et al. (2007): "Tracing syndromespecific trajectories of attention throughout life", Cortex, 43(6): 672-685.

Cornish, K. M. et al. (2005): "Theory of mind deficits in children with fragile X syndrome", Journal of Intellectual Disability Research, 49(5): 372-378.

Cornish, K. M. et al. (200I): "Neuropsychological and behavioral profile of attention deficits in fragile X syndrome”, Revista Neurologia Clínica, 33(I): 24-29.

Dagnan, D. \& Jahoda, A. (2006): “Cognitivebehavioural intervention for people with intellectual disability and anxiety disorders", Journal of Applied Research in Intellectual Disabilities, I9(I): 9I-97.

De Diego Otero, Y. (20I4): "Propuestas de intervención multidisciplinar integrada para el Síndrome X frágil”. En: Medina, B. et al. (coords.): Sindrome X frágil. Manual para familias y profesionales, Tarragona: Publicaciones Altaria.

De Diego Otero, Y. et al. (2009): “Alphatocopherol protects against oxidative stress in the fragile X knockout mouse: an experimental therapeutic approach for the Fmr I deficiency", Neuropsychologypharmacology, 34(4): IOI I-IO26. 
Diez-Itza, E. et al. (20I4): “Lenguaje y comunicación en el síndrome X frágil”. En: Medina, B. et al. (coords.): Sindrome X frágil. Manual para familias y profesionales, Tarragona: Publicaciones Altaria.

Eliez, S. et al. (200I): "Brain anatomy, gender and IQ in children and adolescents with fragile $\mathrm{X}$ syndrome", Brain, I24(8): I6IO-I6I8.

Fernández-Carvajal, I. (20I4): "Nuevas patologías causadas por la premutación”. En: Medina, B. et al. (coords.): Síndrome X frágil. Manual para familias y profesionales, Tarragona: Publicaciones Altaria.

Fernández-Carvajal, I. y De Diego, Y. (20I4): "Técnicas de diagnóstico y prevención. En: Medina, B. et al. (coords.): Sindrome X frágil. Manual para familias y profesionales, Tarragona: Publicaciones Altaria.

Fernández-Carvajal, I. et al. (2009): "Screening for expanded alleles of the FMRI gene in blood spots from newborn males in a Spanish population”, Journal Molecular Diagnostic, II (4): 324-329.

Fernández, M. P. et al. (2010): "Rasgos conductuales y cognitivos de los síndromes Rett, Cri-du-Chat, X-frágil y Williams”, Liberabit, I6: I.

Ferrando, M. T. y Puente, A. (2008): “Niñas con síndrome X frágil: un modelo para los trastornos específicos del desarrollo", Revista Neurología Clínica, 46: I7-I9.

Ferrando, M. T. et al. (2004): “Aspectos cognitivos en niñas con X frágil”, Revista Neurología Clínica, 38 (S.I): 53-58.

Franco, V. (2013): Síndrome de X frágil: pessoas, contextos e percursos, Évora: Aloendro.

Fu, Y. H. et al. (I99I): "Variation of the CGG repeat at the fragile $\mathrm{X}$ site results in genetic instability: resolution of the Sherman paradox", Cell, 67(6): I047-I058.

Garber, K. B. et al. (2008): "Fragile X syndrome”, European Journal Human Genetics, I6: 666-672.

García Alonso, M. I. et al. (2008): "Estudio sobre la prevalencia del síndrome $\mathrm{x}$ frágil en la población de varones adultos atendidos en los centros de discapacitados intelectuales de Burgos. De la conducta a la genética”, International Journal of Developmental and Educational Psychology, I: I07-I I6.

García-Nonell, C. et al. (2006): “Autismo en el síndrome X frágil”, Revista Neurologia Clínica, 42(S2): 95-98.

Glover-López, G. y Guillen, E. (2006): “Síndrome X frágil”, Revista Neurología Clínica, $42\left(\mathrm{~S}_{\mathrm{I}}\right)$ : $5 \mathrm{I}-54$.

Grangreco, C. et al. (1996): “A simplified six item checklist for screening for fragile $\mathrm{x}$ syndrome in the pediatric population", Journal Pediatric, I 29: 6II-6I5.

Greco, C. M. et al. (2002): "Neuronal intranuclear inclusions in a new cerebellar tremor/ataxia syndrome among fragile X carriers", Brian, I 25: I760-I77I.

Hagerman, R. J. et al. (2009): "Advances in the Treatment of Fragile X Syndrome”, Pediatrics, I 23 ( I):378-390 (en línea). 〈doi:IO.I 542/ peds.2008-03I7'.

Hagerman, R. J. (2008): “The fragile X prevalence paradox", Journal of Medical Genetics, 45: 498-499.

Hagerman, R. J. (2004): "Physical and behavioural characteristics of fragile X syndrome”. En: Dew-Huges, D. (ed.): Educating children with Fragile X Syndrome, New York: Routledge.

Hagerman, R. J. y Cronister, A. (1996): Fragile X syndrome:diagnosis, treatment and research, Baltimore, MD: The John Hopkins University Press.

Hagerman, R. J. et al. (I99I): "Fragile X Checklist", American Journal Human Genetic, 38: $283-287$.

Hall, S. S. et al. (2009): "Using percentile schedules to increase eye contact in children with fragile X syndrome", Journal of Applied Behavior Analysis, 42(I): I7I-I76.

Hatton, D. D.et al. (2002): "Problem behavior in boys with fragile X syndrome", American Journal of Medical Genetics, IO8(2): I05-I I6 (en línea). 〈doi:I0.I002/ajmg.IO2I6〉. 
Hodapp, R. M. y Dykens, E. M. (2004): “Genética y fenotipo conductual en las discapacidad intelectual: Su aplicación a la cognición y a la conducta problemática ( $\mathrm{I}^{\circ}$ parte)", Revista Síndrome de Down, 21: I34-I49 (en línea). http://www.downcantabria.com/ revistapdf/83/I 34 -I 49 .pdf .

Hoeft, F. et al. (2010): "Region- specific alterations in brain development in one-to three-yearold boys with fragile X syndrome", PNAS, I07(20): 9335-9339.

Kates, W. R. et al. (I997): "Reliability and validity of MRI measurement of the amygdala and hippocampus in children with fragile X syndrome”, Psychiatry Research Neuroimagen, $75(\mathrm{I}): 3 \mathrm{I}-48$.

Kaufmann, W. E. et al. (2004): “Autism spectrum disorder in fragile $\mathrm{X}$ syndrome. Comunication, Social interaction, and specific behaviors", American Journal of Medical Genetic, I29a: 225-238.

Kittlerof, P. M. et al. (2008): "Dual task processing as a measure of executive function.a comparison between adults with Williams and Down and Down syndrome", American Journal Mental Retardation, II3: I I7-I32.

Losh, M. et al. (2OI2): "Social communication and theory of mind in boys with autism and fragile $\mathrm{X}$ syndrome”, Frontiers in psychology, 3.

Lubs, H. A. (1969): “A marker X chromosome”, American Journal Human Genetic, 2I (3): $23 \mathrm{I}-244$.

Maes, B. et al. (2000): "Phenotypic checklist to screen for fragile X syndrome in people with mental retardation", Mental Retardation, $38(3): 207-2$ I 5 .

Mallolas, J. et al. (2000): "Estudio clínico, bioquímico, neurológico y molecular de I I pacientes que presentan mutaciones nuevas en el gen PAH”, Revista Neurologia Clínica, 3I(IO): 907-9IO.

Martin-Pérez, C. et al. (2003): "Prevalencia de psicopatías en un centro de salud rural”, Atención Primaria, 31: 39-46.

Martínez, F. (2006): “Genética y herencia”. En: Tejada, M. I. (dir.): Síndrome X frágil. Libro de consulta para familias profesionales, Madrid: Real Patronato sobre Discapacidad (en línea). http://www.fraxa.org/pdf/librosxf.pdf).

McDuffe, A. et al. (2010): "Autism spectrum disorder in children and adolescents with fragile X syndrome: within-syndrome differences and age-related changes", American Journal on Intellectual and Developmental Disabilities, I I 5(4): 307-326.

Medina-Gómez, B. (20I4): "Fenotipo conductual: conocer para comprender". En: Medina, B. et al. (coords.): Síndrome X frágil. Manual para familias y profesionales, Tarragona: Publicaciones Altaria.

Menon, V. et al. (2004): "Frontostriatal deficits in fragile X syndrome: Relation to FMR I gene expression”, PNAS, Iог(го): 361 5-3620 (en línea). 〈doiro.I073pnas.0304544IOI〉.

Mínguez, M. et al. (2008): "Risk of cognitive impairment in female premutation carriers of fragile X premutation: analysis by means of robust segmented linear regression models", American Journal Medical Genetic, $\mathrm{I} 5 \circ \mathrm{B}(2)$ : 262-270.

Mostofsky, S. H. et al. (I998): "Evaluation of Cerebellar Size in Attention-Deficit Hyperactivity Disorder", Journal Children Neurology, I3: 434-439 (en línea). ‘doi:Io.I I 7 7/088307389801300904Moà.

Munir, F. et al. (2000): "Nature of the working memory deficit in fragile X syndrome", Brain and Cognition, 44:387-340 (en línea). 〈doi:I0.I006/brcg.I999.I 200〉.

Novel, R. y Estrada, S. (20I4): "Psicopatología y fenotipo cognitivo conductual en las personas con síndrome X frágil”. En: Medina, B. et al. (coords.): Sindrome X frágil. Manual para familias y profesionales, Tarragona: Publicaciones Altaria.

Peng, D. X. et al. (2013): "Cognitive and behavioral correlates of caudate subregion shape variation in fragile X syndrome", Human Brain Mapping, 35(6): 286I-2868 (en línea). 〈doi:IO.I002/hbm. 22376〉.

Ramos, F. (2006): "Fenotipo físico y manifestaciones clínicas”. En: Tejada, I. 
(dir): Sindrome X Frágil. Libro de consulta para familias y profesionales, Madrid: Real Patronato sobre Discapacidad (en línea). http:// www.fraxa.org/pdf/librosxf.pdf».

Reddy, K. S. (2005): “Cytogenetic abnormalities and fragile-X syndrome in Autism Spectrum Disorder”, Medical Genetics, 6(I): 3 (en línea). 〈doi:IO.II 86/I47I-2350-6-3〉.

Rivera, S. M. et al. (2002): "Functional brain activation during arithmetic processing in females with fragile $\mathrm{X}$ syndrome is related to FMR I protein expression", Human Brain Mapping, I6(4): 206-2I8.

Rodríguez-Revenga, L. et al. (2009): "Penetrance of FMR I premutation associated pathologies in fragile X syndrome families”, European Journal of Human Genetics, I7: I359-I362.

Romero-Zerbo, Y. et al. (2009): "Protective effects of melatonin against oxidative stress in Fmr I knockout mice: a therapeutic research model for the fragile X syndrome”, Journal Pineal Research, 46(2): 224-234.

Rosso, T. R. F. \& Darnells, B. V. (20I2): "Contagem numérica em estudantes com síndromes de X-Frágil e Prader-Willi”, Revista brasileira de educação especial, I 8(2): 23 I-244.

Saen-Oon, S. et al. (2008): "Remote mutations and active site dynamics correlate with catalytic properties of purine nucleoside phosphorylase", Biophysical Journal, 94(го): 4078-4088.

Scharfenaker, S. (I999): “Características del habla y del lenguaje en el síndrome X frágil”. En: Ramos-Fuentes, F. (dir.): El sindrome $x$ frágil. Material educativo de la fundación Nacional del $x$ frágil de Estados Unidos, Madrid: Ministerio de Trabajo y Asuntos Sociales.

Song, H. R. et al. (2003): "Identification of protein mutations cartilage oligomeric matrix (COMP) gene in patients with multiple epiphyseal dysplasia and pseudoachondroplasia”, Journal of Human Genetics, 48(5): 222-225.

Symons, F. J. et al. (2010): "Self-injurious behavior in young boys with fragile syndrome", American Journal of Medical Genetic, I I 8(2): I I -I 2 I.

Tejada, M. I. et al. (2008): "Analysis of the molecular parameters that could predict the risk of manifesting premature ovarian failure in female premutation carriers of fragile $\mathrm{X}$ syndrome", Menopause, I 5 (5): 945-949.

Toft, M. et al. (2005): "Parkinsonism, FXTAS, and $F M R I$ premutations”, Movement Disorders, 20:230-233.

Turk, J. (I995): “Fragile X syndrome”, Archives Disease Childhood, 72(I): 3-5.

Van der Molen, M. J. W. et al. (2010): "Profiling fragile $\mathrm{X}$ syndrome in males: strengths and weaknesses in cognitive abilities", Research in Developmental Disabilities, 3 I: 426-439.

Verkerk, A. J. et al. (I99I): "Identification of a gene (FMR-I) containing a CGG repeat coincident with a breakpoint cluster region exhibiting length variation in fragile $\mathrm{X}$ syndrome”, Cell, 65: 905-914.

Warren, S. T. \& Sherman, S. L. (200I): “The fragile X syndrome", The Metabolic and Molecular Bases of Inherited Disease, I: I257-I 290.

Westmar, C. J. \& Maller, J. S. (2007): "FMRP mediates $\mathrm{m}$ GluR 5-dependent translation of amyloid precursor protein", Plos Biology, $5(3): 52$.

Wilbarger, J. \& Wilbarger, P. (2002): Clinical application of the sensory diet. Sensory Integration, Theory and Practice, Filadelfia: Bundy.

Wisbeck, J. M. et al. (2000): "Cortisol and Social Stressors in Children with Fragile X: A Pilot Study", Journal of developmental Beh. 